\title{
Correlation of Aggressivity Papilloma Recurrent Respiratory Tract With Human Papillomavirus Types 6 And 11
}

\author{
Rizka Fathoni Perdana ${ }^{1}$, Sri Herawati ${ }^{1}$, Bakti Surarso ${ }^{1}$, E Bimo Aksono H $^{2}$ \\ ${ }^{1}$ Department of Otorhinolaryngology, Faculty of Medicine-Dr. Soetomo General Hospital, Universitas Airlangga, \\ Surabaya 60285, Indonesia, ${ }^{2}$ Institute of Tropical Diseases-Faculty of Veterinary, Universitas Airlangga, Surabaya
}

\begin{abstract}
Background : Recurrent respiratory tract papilloma (PSPB) is a viral disease that correlated with an airway exophthalmia lesion. Papilloma is primarily caused by human papillomavirus (HPV) types 6 and 11 which are classified as low-risk HPV. Reports indicate that patients with the HPV-11 experience more aggressive course of the disease than HPV-6. Patients are often diagnosed at a younger age, the longer duration, require more frequent surgery and rare diseases of the disease when compared with those caused by HPV-6.
\end{abstract}

Objectives : To investigate the correlation of aggressiveness of PSPB with HPV-6 and 11.

Methods : Fifteen samples were conducted in the Outpatient Unit of Otorhinolaryngology and the Lotus Inpatient Installation of Dr. Soetomo General Hospital from December 2012 to March 2013 then determine the disease aggressiveness. PCR examination was done at the Tropical Disease Institute of Universitas Airlangga. Aggressive correlation of disease with HPV type was tested using Chi-Square.

Results : Fifteen patients were enrolled that divided; the male was 10 and female was 5 . The mean annual number of operations was $2.8 \pm 1.27$ and 9 patients reported more than 3 surgeries each year. 10 patients had distal papilloma and 11 patients had a history of tracheostomy. PSPB was found in 12 patients, HPV-11 was 9 patients and HPV-6 was 6 patients. Chi-square test results showed no significant results with $p=0.525$.

Conclusion : There was no correlation between PSPB aggression with HPV-6 and 11

Keywords : Aggressive Papilloma Respiratory Channels, HPV types 6 and 11, Chi Square.

\section{Introduction}

Recurrent respiratory tract papilloma (PSPB) is divided into child-onset PSPB or juvenile type and adult or adult-onset PSPB ${ }^{1,2}$. Recurrent respiratory tract pneumonia is a disease caused by HPV in particular types 6 and $11^{3,4}$. The manifestation of this viral infection is an esophageal lesion in the respiratory tract and has repetitive growth properties. This disease might undergo remission after obtaining multiple microscopic laryngeal surgeries, but in other conditions, it continues to grow and spread along the respiratory tract making it

\section{Corresponding author:}

Department of Otorhinolaryngology, Faculty of Medicine-Dr. Soetomo General Hospital, Universitas Airlangga, Surabaya 60285, Indonesia more difficult to cure ${ }^{5,6}$.

There are $76.47 \%$ of patients with implantation of the distal papilloma, even $21.57 \%$ has expanded to the trachea and bronchi. The tracheotomy was performed in $60.78 \%$ and $10 \%$ were tracheotomized more than once. A history of microscopic laryngeal surgery three times or more was obtained in $14.58 \%$ of patients. The transition sites include the inferior surface of the vocal cord, the vestibule of the rice, the nasopharyngeal mole palate, the trachea, the carina, and the bronchi, as well as the area around the tracheotomy stoma ${ }^{7}$. Virus type examination could predict the prognosis of disease travel ${ }^{8}$.

Alternative therapies began to be considered based on the course of the disease. In-study therapy was included interferon- $\alpha$, indole- 3 -carbinol, retinoid, bevacizumab, and cidofovir ${ }^{9}$. Human papillomavirus 
(HPV) part of the cellular-mediated immune-response ${ }^{10}$, a virus belonging to the Papoviridae group that has no capsule, icosahedral, composed of double chains by 8000 pairs of DNA molecular bases ${ }^{11,12}$. There were 35 types that infect the genital mucosa, including high-risk types correlated with malignancy and low risk with condyloma or low-grade cervical intraepithelial neoplasia ${ }^{12}$. Human papillomavirus is considered an etiologic factor in PSPB with the discovery of an antigen or genome of HPV in papilloma tissue ${ }^{13}$.

Mounts, et al (1982) examined capsid antigen in the surface epithelial nucleus that using immunoperoxidase techniques and the HPV genome in papillary tumor tissue was using hybridization analysis techniques. Papillary viral antigens were found in four of the 20 PSPB specimens, while the HPV genome was found in all specimens ${ }^{13}$. HPV transmission is suspected to be multifactorial, but in children, the most common risk factor is vertical transmission when the delivery from an HPV-infected mother. There was an increased risk of juvenile PSPB up to 200 times in women with genital condylomata accuminata. Transmission of adult type PSPB occurs through oral sexual activity with multiple partners ${ }^{14}$. Cesarean section operation is estimated to decrease the incidence of vertical HPV transmission ${ }^{15}$.

Recurrent respiratory pneumonia is a problem for the THTKL expert because of the unknown aggressiveness correlation with HPV-6 and 11. This study intends to identify HPV types 6 and 11 in patients with PSPB is expected to be a factor in predicting the aggressiveness of the disease.

\section{Method}

An observational cross-sectional analytic was used by observing how HPV-6 and 11 in recurrent respiratory tract could lead to aggressive or non-aggressive disease $^{16}$. Aggressive PSPB criteria were diseases that require surgical removal of papilloma three or more times within a year, have undergone a tracheotomy, or have implanted distal laryngeal papilloma on rigid or flexible laryngoscopy examination ${ }^{17}$. The nonaggressive PSPB criterion was a disease requiring surgical removal of papilloma less than three times within a year, never undergoing a tracheotomy and no implantation of the distal laryngeal papilloma on rigid or flexible laryngoscopy ${ }^{17}$.

The PSPB patient the Outpatient Otolaryngology Dr. Soetomo General Hospital and undergoing Microscopic Laryngeal Surgery (BLM) at Surgical Installation Center from December 2012 to March 2013. The sample of this study came from the tissue of patients with PSPB who underwent BLM. The inclusion criteria were respiratory papilloma recurrence of all ages, medical records of patients during the complete treatment, and willing to participate the research by signing an informed consent. Patients will be determined by the group aggressive or not aggressive. The tissue of BLM surgery results conducted at Surgical Installation Center of Dr. Soetomo General Hospital was as a research sample. The sample was taken at the Universitas Airlangga Tropical Disease Institute for HPV-6 and 11 examinations using PCR (Polymerase chain reaction) method. The research data was tabulated in the data collection sheet and then processed statistically using the $\mathrm{x} 2$ test.

This research requires several tools in the implementation, that were Microscopic microscopic surgical devices include Optomic OP C-12 optical microscopes, Kleinsasser laryngoscope brand Storz 8590C, Aesculap straight or upturn forceps, cotton, lidocaine-ephedrine $8 \%$, and suction set. The operative specimens were stored in a glass bottle filled with $0.9 \%$ normal saline liquor. Polymerase chain reaction checking tool is with PCR Thermal Cycler engine from Bioneer, HPV Intron $2 x$ primer reagent PCR master mix. Primary HPV-6 in LCR region along 258-361 base pairs in the 5'-TAG GGG ACG GTC CTC TAT TC-3 'or 5'-GCA ACA GCC TCT GAG TCA CA-3' sequence. While the HPV-11 primer at the L1 region along 356 base pairs with the base sequence of 5'-GAA TAC ATG CGC CAT GTG GA-3 'or 5'-AGC AGA CGT CCG TCC TCG AT-3'.

\section{Results}

There were 15 patients that all of them was a child of PSPB type. All patients had complete data during the first diagnosis up to the control so that all were included in the study 
Table 1. Distribution of distal laryngeal papilloma and history of tracheotomy of PSPB patients in Dr. Soetomo General Hospital Surabaya in December 2012 to March 2013

\begin{tabular}{|l|l|l|}
\hline & Amount & Percentase \\
\hline Distal laryngeal lymphoma & & \\
Yes & 10 & 66.67 \\
No & 5 & 33.33 \\
History of tracheotomy & 11 & 73.33 \\
Yes & 4 & 26.67 \\
No & & \\
\hline
\end{tabular}

Distal laryngeal lymphoma was found in 10 patients (66.67\%), while 5 patients $(33.33 \%)$ had a history of papilloma in the distal larynx. Tracheotomy history was found for 11 patients $(73.33 \%)$, while 4 patients $(26.67 \%)$ did not have a history of tracheotomy.

Table 2. Distribution of PSPB aggressiveness of PSPB patients in Dr. Soetomo General Hospital Surabaya in December 2012 to March 2013

\begin{tabular}{|l|l|l|}
\hline Aggressiveness & Amount & Percentase \\
\hline & & \\
Aggressive & 12 & 80 \\
Non-aggressive & 3 & 20 \\
& & \\
\hline
\end{tabular}

Aggressive papilloma was obtained from 12 patients (80\%), while 3 patients (20\%) were classified as non-aggressive PSPB.

Table 3 Distribution of HPV-6 and 11 patients with PSPB in Dr. Soetomo General Hospital Surabaya in December 2012 to March 2013

\begin{tabular}{|l|l|l|}
\hline HPV Type & Amount & Percentase \\
\hline HPV-6 & 6 & 40 \\
HPV-11 & 9 & 60 \\
\hline
\end{tabular}

Human papillomavirus type 6 was obtained in 6 patients (40\%) and $9(60 \%)$ were infected with HPV-11.

Table 4. Distribution of annual BLM history, distal laryngeal palsy, and history of tracheotomy on HPV-6 and 11 patients with PSPB in Dr. Soetomo General Hospital Surabaya in December 2012 to March 2013

\begin{tabular}{|l|l|l|l|}
\hline \multirow{2}{*}{} & \multicolumn{2}{|l|}{ HPV Types } & \multirow{2}{*}{ Total } \\
\cline { 2 - 3 } & $\mathbf{6}$ & $\mathbf{1 1}$ & \\
\hline $\begin{array}{l}\text { History of BLM every year } \\
<3 \mathrm{x} \\
\geq 3 \mathrm{x}\end{array}$ & $\begin{array}{l}3(50 \%) \\
3(33.33 \%)\end{array}$ & $\begin{array}{l}3(50 \%) \\
6(66.67 \%)\end{array}$ & $\begin{array}{l}6(100 \%) \\
9(100 \%)\end{array}$ \\
\hline $\begin{array}{l}\text { Distal laryngeal lymphoma } \\
\text { Yes }\end{array}$ & $3(30 \%)$ & $7(70 \%)$ & $10(100 \%)$ \\
No & $3(60 \%)$ & $2(40 \%)$ & $5(100 \%)$ \\
\hline History of tracheotomy & & & \\
Yes & $4(36.36 \%)$ & $7(63.63 \%)$ & $11(100 \%)$ \\
No & $2(50 \%)$ & $2(50 \%)$ & $4(100 \%)$ \\
\hline
\end{tabular}


The history of BLM $<3 \mathrm{x}$ was obtained in three patients (50\%) with HPV-6 and HPV-11. Whereas the history of BLM $\geq 3 \mathrm{x}$ was obtained by 3 patients $(33.33 \%)$ with HPV-6 and 6 samples (66.67\%) with HPV-11. The history of distal laryngeal papilloma was obtained in 3 patients (30\%) with HPV-6 and 7 patients $(70 \%)$ with HPV-11. Patients without the history of distal laryngeal papilloma consisted of 3 patients $(60 \%)$ with HPV6 and 2 patients (40\%) with HPV-11. The history of tracheotomy was found in 4 patients $(36.36 \%)$ in HPV6 and 7 patients $(63.64 \%)$ in HPV-11. Patients without a history of tracheotomy have the same amount. i.e., 2 patients (50\%) in both HPV-6 and HPV-11 (Table 10).

Table 5 Aggressive correlation between PSPB and HPV-6 and 11 patients with PSPB at Dr. Soetomo General Hospital Surabaya in December 2012 to March 2013

\begin{tabular}{|l|l|l|l|}
\hline & \multicolumn{2}{|l|}{ HPV types } & \multirow{2}{*}{ Fisher } \\
\cline { 2 - 4 } & $\mathbf{6}$ & $\mathbf{1 1}$ & \\
\hline PSPB agrressivity & $4(33.33 \%)$ & $8(66.67 \%)$ & 0.525 \\
Aggressive & $2(66.67 \%)$ & $1(33.33 \%)$ & \\
Non-aggressive & & & \\
\hline
\end{tabular}

Aggressive recurrent respiratory febrile respiration consists of 4 patients with HPV-6 (33.33\% of all HPV6 patients) and 8 patients with HPV-11 (66.67\% of all HPV-11 sufferers). Recurrent non-aggressive respiratory nasal passages consisted of $2 \mathrm{HPV}-6$ patients $(66.67 \%$ of all HPV-6 patients) and 1 patient (33.33\% of all HPV11 sufferers). One-Sample Kolmogorov-Smirnov test results obtained $\mathrm{p}=0.002$. Fisher test results obtained $\mathrm{p}$ $=0.525($ Table 11$)$.

\section{Discussion}

Table 1 shows the percentage of papilloma growing at the distal site of the larynx was $66.7 \%$ and the tracheotomy history was $73.3 \%$. It shows that most sufferers have aggressive disease. The figure was proportional to Doyle. et al. (1994) that reported $60 \%$ had a history of tracheotomy and $55 \%$ had distal papilloma ${ }^{18}$. Wiatrak et al. (2004) reported $11 \%$ had the tracheotomy and $23.3 \%$ experienced distal papilloma growth $^{19}$. The more aggressive course of the disease was seen from the many incidences of distal papilloma growth and the history of tracheotomy.

Table 2 presents an aggressive disease was $80 \%$. Some of the aggressive PSPB findings was performed by Doyle. et al. (1994) with the amount $58.8 \%$ and Buchinsky. et al. (2008) was $81 \%$. The results were in accordance with the above findings. The high findings of the aggressive PSPB were consistent with the high incidence of aggressive factors in the above discussion. All patients were classified as juvenile type $\mathrm{PSPB}^{17,18}$.
Table 3 shows the percentage of HPV-6 infections by $40 \%$ and HPV-11 by $60 \%$. No samples with double infection or HPV infection other than types 6 and 11. Wiatrak. et al. (2004) stated that patients with HPV-6 by $53.5 \%$, HPV-11 was $39.7 \%$ and $6.9 \%$ had both types. Buchinsky. et al. (2008) get the percentage of HPV-6 infection by $60 \%$ and HPV- -11 by $40 \%$. The results of this study were in accordance with Buchinsky that the incidence of HPV-11 infection was more dominant than HPV $-6^{17}$.

Table 4 was the distribution of annual BLM history correlation, distal papilloma and tracheotomy history of HPV-6 and 11. The annual BLM history was grouped into two categories i.e., BLM history of more or equal to $3 \mathrm{x}(\geq 3 \mathrm{x})$ within one year and BLM history less than $3 \mathrm{x}$ within one year period provided that the BLM history $\geq$ $3 \mathrm{x}$ was an aggressive type of PSPB category.

Patients with a history of BLM every year $<3 \mathrm{x}$ consisted of 3 persons each with HPV-6 and HPV-11 infections, while patients with annual BLM category $\geq 3 \mathrm{x}$ consisted of 3 people with HPV-6 and 6 people with HPV-11. Human papillomavirus type 11 causes the patient to experience BLM every year $\geq 3 \mathrm{x}$ more often than BLM each year $<3 x$ ( 6 samples vs. 3 samples), while HPV- 6 balanced in causing BLM every year $\geq 3 \mathrm{x}$ or $<3 \mathrm{x}$. Human papillomavirus type 11 tends to cause BLM surgery more frequently.

Papilloma with spread to the distal larynx was one indicator of disease aggressiveness. Seven patients with distal laryngeal palsy are known to be infected with 
HPV-11, while a number of 3 patients infected with HPV-6. Patients with no history of distal laryngeal papilloma accompanied by HPV-6 were 3 patients and 2 patients with HPV-11. Wiatrak. et al. (2004) stated that 12 samples for papilloma in the trachea with HPV-11 infection and 11 patients with HPV-6. Patients without a history of papilloma in the trachea consisted of 28 patients with HPV-6 and 11 patients with HPV-11 19 . This study was in accordance with the literature that HPV-11 tends to cause patients to have distal laryngeal palsy compared with HPV-6.

Seven patients with a history of tracheotomy had the HPV-11 infection and 4 HPV-6 infected persons, while patients without tracheotomy obtained 2 patients with HPV-6 infection and HPV-11. Wiatrak. et al. (2004) stated that $21.7 \%$ of patients with a history of tracheotomy having HPV-11 of 5 samples and 1 sample with HPV-6. Patients without a history of tracheotomy consisted of 30 samples with HPV-6 and 18 samples with HPV $-11^{19}$. The results were similar to the literature findings because the trends in HPV-11 cause the patient to require tracheotomy compared with HPV-6.

Table 5 shows the correlation between aggressiveness and HPV types 6 and 11. The assessment aspect of the correlation was whether there was an association between aggressive PSPB and HPV-11 versus non-aggressive PSPB with HPV-6. Aggressive respiratory febrile respiratory infections were $8 \mathrm{HPV}$ 11 infected and 4 with HPV-6, while the non-aggressive PSPB consisted of 2 HPV-6 and 1 patients with HPV-6.

The results of the One-Sample KolmogorovSmirnov test was $p=0.002$ which mean the data has abnormal distribution because of the value of $p<0.05$. Fisher test got result $\mathrm{p}=0.525$ meaning that there was no correlation between aggressiveness PSPB and HPV types 6 and 11 because of $p>0.05$.

Buchinsky. et al. (2008) obtained 43 aggressive PSPB samples with HPV-11 infection and 52 patients with HPV-6, while PSPB was not aggressive with HPV6 infection of 19 patients and 4 patients with HPV-11. These results indicate that HPV-11 tends to cause more aggressive disease than HPV-6 and the correlation was statistically significant with $\mathrm{p}=0.02(\mathrm{p}<0.05)$.

\section{Conclusion}

There was no corrrelation between recurrent respiratory tract aggressiveness with HPV-6 and 11.
More aggressive recurrent respiratory papillomas more infected with HPV-11 and HPV-6 than recurrent nonaggressive respiratory tract papillomas.

Ethical Clearance: The present study was carried out in accordance with the research principles. This study implemented the basic principle ethics of respect, beneficence, nonmaleficence, and justice.

Conflict of Interest: None declared

Source of Funding: This study is done with individual funding.

\section{Referrence}

1. Larson DA, Derkay CS. Epidemiology of recurrent respiratory papillomatosis. Apmis. 2010;118(6-7):450-4.

2. Derkay CS. Recurrent respiratory papillomatosis. The Laryngoscope. 2001;111(1):57-69.

3. Agus P. Prognostic value of molecular markers of oral pre-malignant and malignant lesions. Dental Journal (Majalah Kedokteran Gigi). 2009;42(2):104-8.

4. Perdana RF, Herawati S, Suroso B, Aksono EB. no ASSociAtion of rEcurrEnt rESPirAtory PAPillomAtoSiS AggrESSivEnESS And HumAn PAPillomAviruS tyPE 6 And 11. Indonesian Journal of Tropical and Infectious Disease. 2017;6(5):113-7.

5. Lee JH, Smith RJ. Recurrent respiratory papillomatosis: pathogenesis to treatment. Current opinion in otolaryngology $\&$ head and neck surgery. 2005;13(6):354-9.

6. Freed GL, Derkay CS. Prevention of recurrent respiratory papillomatosis: role of HPV vaccination. International journal of pediatric otorhinolaryngology. 2006;70(10):1799-803.

7. Donne AJ, Clarke R. Recurrent respiratory papillomatosis: an uncommon but potentially devastating effect of human papillomavirus in children. International journal of STD \& AIDS. 2010;21(6):381-5.

8. Derkay CS, Darrow DH. Seminar Series Recurrent Respiratory Papillomatosis. Annals of Otology, Rhinology \& Laryngology. 2006;115(1):1-11.

9. Decherd SM. Primary Productivity and Forage Quality of Gingko Biloba in Response to Elevated Carbon Dioxide and Oxygen-An Experimental 
Approach to Mid-mesozoic Paleoecology. 2006;

10. FatimahN,FarmakologiD.PHARMACOLOGICAL ASPECT OF ANTI HERPETIC DRUGS.

11. Van Ranst M, Kaplan JB, Burk RD. Phylogenetic classification of human papillomaviruses: correlation with clinical manifestations. Journal of General Virology. 1992;73(10):2653-60.

12. Molijn A, Kleter B, Quint W, van Doorn L-J. Molecular diagnosis of human papillomavirus (HPV) infections. Journal of clinical virology. 2005;32:43-51.

13. Mounts P, Kashima H. Association of human papillomavirus subtype and clinical course in respiratory papillomatosis. The Laryngoscope. 1984;94(1):28-33.

14. Goon P, Sonnex C, Jani P, Stanley M, Sudhoff H. Recurrentrespiratory papillomatosis: an overview of current thinking and treatment. European Archives of Oto-Rhino-Laryngology. 2008;265(2):147-51.

15. Kosko JR, Derkay CS. Role of cesarean section in prevention of recurrent respiratory papillomatosisis there one? International journal of pediatric otorhinolaryngology. 1996;35(1):31-8.
16. Nugraha AP, Ernawati DS, Endah PA, Soebadi B, Triyono EA, Prasetyo RA, et al. Correlation Linear Gingival Erythema, Candida Infection and CD4+ Counts in HIV/AIDS Patients at UPIPI RSUD Dr. Soetomo Surabaya, East Java, Indonesia. Journal of International Dental and Medical Research. 2017;10(2):322.

17. Buchinsky FJ, Donfack J, Derkay CS, Choi SS, Conley SF, Myer III CM, et al. Age of child, more than HPV type, is associated with clinical course in recurrent respiratory papillomatosis. PloS one. 2008;3(5):e2263.

18. Doyle DJ, Gianoli GJ, Espinola T, Miller RH. Recurrent respiratory papillomatosis: juvenile versus adult forms. The Laryngoscope. 1994;104(5):523-7.

19. Wiatrak BJ, Wiatrak DW, Broker TR, Lewis L. Recurrent respiratory papillomatosis: a longitudinal study comparing severity associated with human papilloma viral types 6 and 11 and other risk factors in a large pediatric population. The Laryngoscope. 2004;114(S104):1-23. 